\title{
A high eating frequency is associated with an overall healthy lifestyle in middle-aged men and women and reduced likelihood of general and central obesity in men
}

\author{
Isabel Holmbäck*, Ulrika Ericson, Bo Gullberg and Elisabet Wirfält \\ Department of Clinical Sciences in Malmö, Lund University, Malmö, Sweden \\ (Received 9 September 2009 - Revised 11 March 2010 - Accepted 30 March 2010 - First published online 26 May 2010)
}

The role of eating frequency in obesity development is debated. Therefore, we investigated the association between eating frequency, BMI and waist circumference (WC), as well as how eating frequency is related to diet composition and lifestyle factors. A subsample (aged 47-68 years) of men ( $n$ 1355) and women ( $n$ 1654) from the Malmö Diet and Cancer cohort was used for the present cross-sectional study. The daily eating frequency was calculated based on the number of self-reported eating occasions during an ordinary day. Regression analysis and ANOVA examined the associations between eating frequency, BMI and WC, while adjusting for potential confounders. The energy percentage (E\%) from carbohydrates as well as relative fibre intake $(\mathrm{g} / \mathrm{MJ})$ increased with higher eating frequency; while E\% from fat, protein and alcohol decreased. A low daily eating frequency was associated with smoking, higher alcohol consumption, and lower leisure-time physical activity. Eating three or fewer meals per d was also associated with increased likelihood of general and central obesity in men when adjusting for total energy intake, lifestyle and dietary factors. However, results did not reach statistical significance among women. The present study suggests that a high daily eating frequency is associated with a healthy lifestyle and dietary pattern in both men and women, and a reduced likelihood of general and central obesity in men. There is a need for prospective studies investigating the association between eating frequency, diet and body composition.

Eating frequency: BMI: Waist circumference: Obesity: Lifestyle: Diet quality

Most researchers agree that the current obesity epidemic predominately results from the combination of energy-dense diets and sedentary lifestyles ${ }^{(1-4)}$. The obesity trend is thus considered to be caused by a combination of factors, and is found in most social groups, in both men and women of all ages ${ }^{(5-8)}$. However, information about specific factors contributing to weight gain and the development of obesity seems to be either inconclusive or contradictory, primarily since most factors are inter-related and difficulties arise when attempting to isolate the effect of one single factor ${ }^{(9-12)}$. In addition, due to confounding, it is difficult to estimate the true impact of the various risk factors for weight gain.

Meal and snack patterns, including the daily eating frequency, are thought to influence weight status as well as development of chronic diseases ${ }^{(13-17)}$. However, several authors have disputed the fact that the eating frequency in itself is related to adverse health effects ${ }^{(18-19)}$. The daily eating frequency is likely to be associated with different food choices as well as lifestyle and socio-economic factors; it may thus be accompanied by changes in dietary quality. Overweight and obese individuals have been found to have different eating habits in comparison with normal-weight individuals, including morning anorexia and consumption of larger food amounts in the afternoon and evening as well as a higher eating frequency ${ }^{(14,20-22)}$. In addition, several studies have shown sex differences in eating patterns ${ }^{(14,23,24)}$. Women are often reported to have lower intake of fat, and higher intakes of fruits and vegetables and dietary fibre. However, women are also more likely to find healthy eating more important, and are more likely to restrain their eating behaviour $^{(25)}$.

Sex and several psychosocial and behavioural characteristics are related to energy misreporting, including incomplete recordkeeping by subjects ${ }^{(26)}$. Therefore it is essential to examine the influence of potential confounding factors on self-reported energy and food intake in dietary research. Previous studies investigating diet-disease associations in the Malmö Diet and Cancer (MDC) cohort have shown that it is important to consider past changes in food habits and energy misreporting in sensitivity analysis ${ }^{(27-29)}$.

The aim of the present study was to examine the associations between daily eating frequency, BMI and waist circumference. In order to identify factors that correlate with eating frequency we examined the association between daily eating frequency and the intake of total energy, selected nutrients and food groups and lifestyle factors. In addition, an important part of this investigation was to evaluate the potential confounding associated with possible reporting errors.

Abbreviations: EI, energy intake; E\%, energy percentage; MDC, Malmö Diet and Cancer.

* Corresponding author: Dr Isabel Holmbäck, fax +46 40391 322, email isabel.holmback@med.lu.se 
An improved understanding of the potential health effects of eating frequency as well as the association between eating frequency and diet quality could be important for future public health advice and strategies dealing with the current obesity epidemic.

\section{Subjects and methods}

Study design

This cross-sectional study examined data from the MDC cohort, a population-based prospective study set in Malmö, Sweden. In 1991, the MDC source population was defined as all individuals living in the city of Malmö and born between 1926 and 1945. In May 1995, the cohort was extended to include all women born between 1923 and 1950 and all men born between 1923 and 1945. With this extension, 74138 individuals constituted the source population. Inadequate Swedish language skills and mental incapacity were the only exclusion criteria. The MDC study was conducted according to the guidelines laid down in the Declaration of Helsinki and all procedures involving human subjects were approved by the Ethical Committee at the Medical Faculty, Lund University (LU 51-90). Written informed consent was obtained from all subjects. Details of the recruitment procedures and the cohort are described elsewhere ${ }^{(30,31)}$. Briefly, the participants were invited by mail or advertisements in the local media and primary health-care centres. The participants visited the MDC screening centre twice. During the first visit, participants were instructed on how to register meals in a menu book and how to fill out the questionnaires that were completed at home. Anthropometric measurements and blood collection were conducted on location by nurses. During the second visit (approximately $10 \mathrm{~d}$ later) the questionnaires were checked and a dietary interview was conducted by trained interviewers. In October 1996, when recruitment was closed, 28098 participants had completed all baseline examinations.

\section{Study participants}

The present study includes individuals that joined the MDC study from January 1993 until March 1994. These participants were, in addition to the other diet history information, requested to give a general overview of their eating occasions during an ordinary day. A total of 3009 subjects (1355 men and 1654 women) aged between 47 and 68 years are included in the study.

\section{Eating frequency assessment}

The questionnaire used for assessment of the daily eating frequency included registration of the number and types of meals and snacks eaten during an ordinary day, and how many times per week these meals or snacks usually were eaten. If weekends differed significantly from weekdays the participants were requested to describe two different days (for further details on registration of meals, see Appendix). The self-reported information was carefully checked by trained nutritionists during the diet history interview. Based on the participants' self-reported information on number of eating occasions (including both snacks and meals) during a week, the average daily eating frequency was calculated. In the present study we excluded non-nutrient contributing snacks, i.e. coffee and tea. The eating frequencies were aggregated into: 'three or fewer', 'four to five' or 'six or more' eating occasions per $\mathrm{d}$.

\section{Dietary assessment}

In the MDC study, food and nutrient intakes were assessed using an interview-based modified diet history method. This methodology combines a $7 \mathrm{~d}$ menu book collecting descriptions of prepared meals, nutrient supplements and cold beverages (including alcoholic beverages) with a 168-item dietary questionnaire covering regularly eaten foods other than prepared meals during the previous year. The consistency of the information provided was carefully checked during a 45 min interview so that the questionnaire and menu book did not overlap. The average energy and nutrient intakes were computed by compiling the reported food intake from the two methodologies and applying this information to the MDC Food and Nutrient Database, mainly originating from the database PC Kost2-93 of the National Food Administration in Uppsala, Sweden ${ }^{(32)}$. Data on the validity ${ }^{(33,34)}$ and reproducibility $^{(35)}$ of the method have been published.

\section{Dietary variables}

The present study examined the associations between eating frequency and total energy intake (EI) (MJ), nutrient densities and intake of selected food groups. The energy percentage (E\%) from fat, carbohydrates and protein was computed using non-alcohol energy. Similarly, the micronutrient densities were expressed as intake per MJ of non-alcohol energy. In addition, alcohol was examined both as percentage of total energy and as alcohol habits (categorical variable, see below). The nutrient variables examined in the present study were: fibre (g/MJ), Fe (mg/MJ), Ca (mg/MJ), Mg (mg/MJ), $\beta$-carotene $(\mathrm{mg} / \mathrm{MJ})$, ascorbic acid $(\mathrm{mg} / \mathrm{MJ})$, folate $(\mu \mathrm{g} / \mathrm{MJ})$ and vitamin $\mathrm{E}(\mathrm{mg} / \mathrm{MJ})$. The food groups examined were: fruits and vegetables; meat, eggs and poultry; low-fat meat; fish and shellfish; milk and dairy products; low-fat dairy; bread and cereals; high-fibre bread and cereals; confectionery foods (the sum of sweets, added sugar, jam, and chocolate); soft drinks; cakes and pastries.

\section{Energy misreporting and past food habit change}

Mattisson et al. ${ }^{(27)}$ have previously defined low-, adequateand high-energy reporters in the MDC cohort using the approach described by Goldberg et al. ${ }^{(36)}$ and later refined by Black ${ }^{(37)}$. Energy misreporting was defined as having an EI:BMR ratio outside the $95 \%$ CI limits of the calculated physical activity level. The physical activity level of each individual was carefully calculated using all available information in the MDC cohort. The estimation used information from the questionnaire concerning hours and intensity of leisure-time physical activity, hours of household work, and hours and intensity of occupational activities. Hours of sleeping, time for self-care, and 'passive' time were estimated ${ }^{(27)}$. Individuals with EI:BMR below the lower $95 \%$ confidence 
limit were classified as under-reporters of EI. Individuals with EI:BMR within the confidence limits were classified as adequate-energy reporters and individuals with EI:BMR above the upper $95 \%$ confidence limit were classified as over-reporters of EI.

In addition, past food habit change was dichotomised (yes or no) based on the questionnaire item 'Have you substantially changed your dietary habits because of illness or another reason?'

\section{Socio-economic and lifestyle variables}

Information on socio-economic and lifestyle factors was collected from the structured lifestyle questionnaire. The participants were divided into four categories to describe educational level according to the number of years of education completed (i.e. $\leq 8$ years, 9-10 years, 11-13 years, or $>13$ years). Socio-economic status was classified according to the Swedish population census as 'blue-collar workers', 'white-collar workers' and 'employers/selfemployed' (in the present study including high-positioned white-collar workers) ${ }^{(38)}$. Leisure-time physical activity was assessed by questions adapted from the Minnesota Leisure Time Physical Activity Questionnaire ${ }^{(39,40)}$. The overall leisure-time physical activity score was categorised into quartiles based on the population distribution. The smoking habits of the participants were defined as current smokers (including irregular smokers), former smokers or neversmokers. Alcohol habits were classified as zero, low, medium or high consumption. Participants reporting no consumption of alcohol during the previous year in the socio-economic and lifestyle questionnaire and that in addition did not report any alcohol intake in the $7 \mathrm{~d}$ menu book were classified as zero alcohol consumers. For all other participants low, medium and high alcohol consumption level was set at alcohol intakes of $<15,15-30$, and $>30 \mathrm{~g} / \mathrm{d}$ for women, and at $<20,20-40$, and $>40 \mathrm{~g} / \mathrm{d}$ for men, based on the information collected in the menu book.

\section{Anthropometric variables}

Nurses measured weight, height and waist circumference. BMI was calculated from weight $(\mathrm{kg})$ over the square of height $\left(\mathrm{m}^{2}\right)$. Relative weight categories (i.e. BMI $<18.5$; 18.5 to $<25 ; 25$ to $<30 ; \geq 30 \mathrm{~kg} / \mathrm{m}^{2}$ ) were used to classify individuals as underweight, normal weight, overweight, or obese, according to WHO recommendations ${ }^{(41)}$. Waist circumference was transformed into dichotomised variables to assess central obesity. Waist circumference associated with increased risk of metabolic and CVD was set to $\geq 80 \mathrm{~cm}$ (women) and $\geq 94 \mathrm{~cm}$ (men); waist circumference associated with a greatly increased risk of metabolic and cardiovascular disease was set to $\geq 88 \mathrm{~cm}$ (women) and $\geq 102 \mathrm{~cm}$ (men), according to WHO recommendations ${ }^{(41)}$.

\section{Statistical analysis}

All analyses investigated men and women separately. The study population characteristics were assessed using the $\chi^{2}$ test and ANOVA. Since energy misreporting and past food habit change was differentially associated with eating frequency, further analysis included only men ( $n$ 892) and women ( $n$ 1024) classified as adequate energy reporters and reporting no past food habit change. Total EI and relative nutrient intakes across daily eating frequency were expressed as mean values with their standard errors, and mean differences examined using ANOVA while adjusting for age. Some nutrient variables were log transformed to normalise the distribution before analysis. Differences in intake from

Table 1. Study population characteristics and differences between men and women

\begin{tabular}{|c|c|c|c|c|}
\hline Characteristics & Total ( $n$ 3009) & Men $(n$ 1355) & Women ( $n$ 1654) & $P$ \\
\hline Age (years) & & & & 0.136 \\
\hline Mean & $57 \cdot 2$ & $57 \cdot 4$ & $57 \cdot 1$ & \\
\hline SD & $6 \cdot 1$ & $6 \cdot 1$ & $6 \cdot 1$ & \\
\hline Energy intake (MJ) & & & & $<0.0001$ \\
\hline Mean & 9.8 & $11 \cdot 3$ & 8.5 & \\
\hline SD & 2.9 & 2.9 & $2 \cdot 1$ & \\
\hline Eating frequency & & & & $<0.0001$ \\
\hline Mean & 4.9 & 4.8 & $5 \cdot 0$ & \\
\hline SD & $1 \cdot 1$ & 1.2 & $1 \cdot 1$ & \\
\hline Leisure-time physical activity score (\%) & & & & 0.080 \\
\hline Quartile 1 & 24.9 & 25.5 & $24 \cdot 3$ & \\
\hline Quartile 2 & 24.9 & $23 \cdot 7$ & 25.9 & \\
\hline Quartile 3 & $25 \cdot 2$ & $24 \cdot 0$ & $26 \cdot 3$ & \\
\hline Quartile 4 & $25 \cdot 0$ & $26 \cdot 8$ & 23.5 & \\
\hline BMI (\%) & & & & $<0.0001$ \\
\hline$<18.5 \mathrm{~kg} / \mathrm{m}^{2}$ & 1.0 & 0.7 & 1.2 & \\
\hline 18.5 to $<25 \mathrm{~kg} / \mathrm{m}^{2}$ & 43.9 & $37 \cdot 1$ & 49.5 & \\
\hline 25 to $<30 \mathrm{~kg} / \mathrm{m}^{2}$ & 41.4 & $47 \cdot 9$ & $36 \cdot 2$ & \\
\hline$\geq 30 \mathrm{~kg} / \mathrm{m}^{2}$ & $13 \cdot 7$ & $14 \cdot 3$ & $13 \cdot 1$ & \\
\hline Waist circumference (\%) & & & & $<0.0001$ \\
\hline Men $<94 ;$ women $<80 \mathrm{~cm}$ & $64 \cdot 1$ & $57 \cdot 6$ & $69 \cdot 3$ & \\
\hline Men 94 to $<102 ;$ women 80 to $<88 \mathrm{~cm}$ & $20 \cdot 3$ & 24.6 & $16 \cdot 8$ & \\
\hline Men $\geq 94 ;$ women $\geq 88 \mathrm{~cm}$ & $15 \cdot 6$ & $17 \cdot 8$ & 13.9 & \\
\hline
\end{tabular}


selected food groups across daily eating frequency were examined using ANOVA adjusting for age and total EI.

The mean differences in BMI and waist circumference across categories of daily eating frequency was examined while adjusting for potential confounders. The risk of overweight/obesity and central obesity associated with the daily eating frequency was estimated using logistic regression with six or more meals per $\mathrm{d}$ as the reference, while adjusting for age, education, socio-economic status, smoking, alcohol consumption, leisure-time physical activity and total EI. Other logistic regression models were in addition adjusted for nutrient and food group variables. OR and $95 \% \mathrm{CI}$ were calculated. Two dichotomous variables identified overweight (i.e. BMI 25 to $<30 \mathrm{~kg} / \mathrm{m}^{2}$ ) and obese (i.e. BMI $\geq 30 \mathrm{~kg} / \mathrm{m}^{2}$ ) individuals respectively, with normal weight (i.e. BMI $18 \cdot 5$ to $<25 \mathrm{~kg} / \mathrm{m}^{2}$ ) as the reference category. The risk associated with a large waist circumference used similar variables (i.e. 94 to $<102$ and $\geq 102 \mathrm{~cm}$ in men; 80 to $<88$ and $\geq 88 \mathrm{~cm}$ in women) using subjects with healthy waist circumferences (i.e. $<94 \mathrm{~cm}$ for men and $<80 \mathrm{~cm}$ for women) as reference. Significance level was set at $P<0 \cdot 05$. The SPSS statistical computer package (version 17; SPSS Inc., Chicago, IL, USA) was used for all statistical analyses.

\section{Results}

The study population characteristics are presented in Table 1 and associations between daily eating frequency and lifestyle and socio-economic factors are presented in Table 2. On average, men reported a daily eating frequency of 4.8 (SD 1.2) with a range of $1-13$ (median $4 \cdot 7$ ). Women reported a daily eating frequency of 5.0 (SD 1.1) with a range of 1-9 (median 5.0). The majority of men and women reported eating between four to five meals per $\mathrm{d}$; however, men were more likely than women to report three or fewer meals per $\mathrm{d}$, while women were more likely to report an eating frequency of six or more meals per $\mathrm{d}(P<0.0001)$. Men and women with a low daily eating frequency (i.e. three or fewer) were more likely to be younger, current smokers, high alcohol consumers, and less physically active compared with subjects reporting a high daily eating frequency (i.e. six or more). These individuals were also more likely to under-report EI, but less likely to have changed their food habits substantially in the past compared with more frequent eaters (Table 2).

The mean relative energy and nutrient intakes across categories of daily eating frequency are shown in Table 3. The analysis excluded energy misreporters and individuals with

Table 2. Study population characteristics across categories of daily eating frequency in men $(n$ 1355) and women $(n$ 1654) (Percentages)

\begin{tabular}{|c|c|c|c|c|c|c|c|c|}
\hline \multirow[b]{2}{*}{ Daily eating frequency... } & \multicolumn{4}{|c|}{ Men } & \multicolumn{4}{|c|}{ Women } \\
\hline & $\begin{array}{c}3 \text { or fewer } \\
(n 181)\end{array}$ & $\begin{array}{c}4 \text { to } 5 \\
(n 824)\end{array}$ & $\begin{array}{l}6 \text { or more } \\
(n 350)\end{array}$ & $P$ & $\begin{array}{c}3 \text { or fewer } \\
(n 134)\end{array}$ & $\begin{array}{l}4 \text { to } 5 \\
(n 382)\end{array}$ & $\begin{array}{l}6 \text { or more } \\
(n 538)\end{array}$ & $P$ \\
\hline All & $13 \cdot 4$ & $60 \cdot 8$ & $25 \cdot 8$ & - & $8 \cdot 1$ & $59 \cdot 4$ & $32 \cdot 5$ & $<0.0001^{*}$ \\
\hline Age & & & & $<0.0001$ & & & & 0.006 \\
\hline$<55$ years & $48 \cdot 1$ & 37.5 & $30 \cdot 6$ & & $52 \cdot 2$ & $40 \cdot 9$ & $35 \cdot 3$ & \\
\hline $55-64$ years & 44.2 & $44 \cdot 3$ & 43.7 & & $36 \cdot \overline{6}$ & $42 \cdot 7$ & $48 \cdot 0$ & \\
\hline$\geq 65$ years & $7 \cdot 7$ & $18 \cdot 2$ & $25 \cdot 7$ & & $11 \cdot 2$ & $16 \cdot 4$ & $16 \cdot 7$ & \\
\hline Education & & & & 0.177 & & & & 0.140 \\
\hline$\leq 8$ years & $40 \cdot 3$ & $46 \cdot 2$ & $51 \cdot 7$ & & 38.1 & 41.6 & $44 \cdot 3$ & \\
\hline $9-10$ years & $18 \cdot 8$ & $20 \cdot 6$ & $16 \cdot 6$ & & $26 \cdot 1$ & $30 \cdot 7$ & $31 \cdot 0$ & \\
\hline $11-13$ years & 14.4 & $12 \cdot 2$ & 11.4 & & $13 \cdot 4$ & 7.9 & 6.5 & \\
\hline$>13$ years & $26 \cdot 5$ & $21 \cdot 0$ & $20 \cdot 3$ & & $22 \cdot 4$ & $19 \cdot 8$ & $18 \cdot 2$ & \\
\hline Socio-economic status & & & & 0.396 & & & & 0.002 \\
\hline Blue-collar & 33.1 & $37 \cdot 7$ & 35.4 & & $44 \cdot 3$ & 39.5 & $40 \cdot 7$ & \\
\hline White-collar (low/medium) & $30 \cdot 9$ & $33 \cdot 7$ & 33.7 & & $38 \cdot 1$ & $45 \cdot 3$ & $50 \cdot 3$ & \\
\hline White-collar (high) and self-employed & $36 \cdot 0$ & $28 \cdot 6$ & $30 \cdot 9$ & & $17 \cdot 6$ & $15 \cdot 2$ & $9 \cdot 0$ & \\
\hline Smoking status & & & & $<0.0001$ & & & & $<0.0001$ \\
\hline Current & 41.4 & $32 \cdot 0$ & $25 \cdot 4$ & & $58 \cdot 3$ & 31.4 & $25 \cdot 0$ & \\
\hline Former & 35.9 & $42 \cdot 0$ & 39.7 & & $15 \cdot 9$ & $27 \cdot 2$ & $24 \cdot 3$ & \\
\hline Never & $22 \cdot 7$ & $26 \cdot 0$ & 34.9 & & $25 \cdot 8$ & $41 . \overline{4}$ & $50 \cdot 7$ & \\
\hline Alcohol consumption & & & & $<0.0001$ & & & & $<0.0001$ \\
\hline Zero & 5.5 & $4 \cdot 1$ & $6 \cdot 0$ & & 7.5 & $7 \cdot 8$ & 8.7 & \\
\hline Low & $50 \cdot 3$ & 65.5 & 68.9 & & 73.9 & $76 \cdot 6$ & $81 \cdot 6$ & \\
\hline Medium & $32 \cdot 0$ & $22 \cdot 6$ & $20 \cdot 3$ & & $10 \cdot 4$ & $13 \cdot 2$ & $9 \cdot 1$ & \\
\hline High & $12 \cdot 2$ & $7 \cdot 8$ & 4.8 & & $8 \cdot 2$ & $2 \cdot 4$ & 0.6 & \\
\hline Leisure-time physical activity score & & & & 0.013 & & & & 0.004 \\
\hline Quartile 1 & $34 \cdot 1$ & $25 \cdot 2$ & $21 \cdot 8$ & & $29 \cdot 8$ & $26 \cdot 3$ & 19.4 & \\
\hline Quartile 2 & $26 \cdot 1$ & 23.9 & $22 \cdot 1$ & & $16 \cdot 7$ & 24.5 & $30 \cdot 7$ & \\
\hline Quartile 3 & $21 \cdot 6$ & $24 \cdot 1$ & $24 \cdot 6$ & & $26 \cdot 0$ & $26 \cdot 1$ & $26 \cdot 7$ & \\
\hline Quartile 4 & $18 \cdot 2$ & $26 \cdot 8$ & 31.5 & & $27 \cdot 5$ & $23 \cdot 1$ & $23 \cdot 2$ & \\
\hline Level of energy reporting & & & & $<0.0001$ & & & & $<0.0001$ \\
\hline Under & $16 \cdot 0$ & $10 \cdot 6$ & 6.6 & & $36 \cdot 6$ & $16 \cdot 7$ & 9.9 & \\
\hline Adequate & $83 \cdot 4$ & $86 \cdot 4$ & $87 \cdot 7$ & & $59 \cdot 7$ & $80 \cdot 8$ & $86 \cdot 8$ & \\
\hline Over & 0.6 & 3.0 & $5 \cdot 7$ & & 3.7 & 2.5 & $3 \cdot 3$ & \\
\hline Past food habit change? & & & & 0.048 & & & & 0.012 \\
\hline Yes & $20 \cdot 4$ & $22 \cdot 3$ & $28 \cdot 3$ & & 23.9 & $22 \cdot 1$ & $29 \cdot 0$ & \\
\hline No & $79 \cdot 6$ & $77 \cdot 7$ & $71 \cdot 7$ & & $76 \cdot 1$ & 77.9 & $71 \cdot 0$ & \\
\hline
\end{tabular}

${ }^{*} x^{2}$ Analysis of sex by eating frequency. 
Table 3. Relative nutrient intakes across categories of daily eating frequency in men ( $n$ 892) and women ( $n$ 1024) adjusting for age $\dagger$ (Mean values with their standard errors)

\begin{tabular}{|c|c|c|c|c|c|c|c|}
\hline & \multicolumn{6}{|c|}{ Daily eating frequency } & \multirow[b]{3}{*}{$P$} \\
\hline & \multicolumn{2}{|c|}{3 or fewer } & \multicolumn{2}{|c|}{4 to 5} & \multicolumn{2}{|c|}{6 or more } & \\
\hline & Mean & SE & Mean & SE & Mean & SE & \\
\hline \multicolumn{8}{|l|}{ Men } \\
\hline Subjects $(n)$ & \multicolumn{2}{|c|}{124} & \multicolumn{2}{|c|}{550} & \multicolumn{2}{|c|}{218} & \\
\hline Total energy intake (MJ) & $10 \cdot 7$ & 0.2 & 11.4 & 0.1 & $12 \cdot 4$ & 0.2 & $<0.0001$ \\
\hline Fat (\% of non-alcohol energy) & $40 \cdot 7$ & 0.5 & $40 \cdot 8$ & 0.3 & $40 \cdot 0$ & 0.4 & 0.290 \\
\hline Carbohydrates (\% of non-alcohol energy) & $43 \cdot 6$ & 0.5 & 43.9 & 0.3 & $45 \cdot 4$ & 0.4 & 0.005 \\
\hline Protein (\% of non-alcohol energy) & $15 \cdot 8$ & 0.2 & $15 \cdot 3$ & 0.1 & $14 \cdot 6$ & 0.2 & $<0.001$ \\
\hline Alcohol ( $\%$ of total energy) & $5 \cdot 8$ & 0.3 & $4 \cdot 1$ & 0.2 & 3.6 & 0.3 & $<0.0001$ \\
\hline Fibre $(g / M J)$ & 1.76 & 0.05 & 1.90 & 0.02 & 2.02 & 0.04 & $<0.0001^{*}$ \\
\hline $\mathrm{Fe}(\mathrm{mg} / \mathrm{MJ})$ & 1.69 & 0.03 & 1.67 & 0.01 & 1.68 & 0.02 & 0.791 \\
\hline $\mathrm{Ca}(\mathrm{mg} / \mathrm{MJ})$ & 104 & 3 & 110 & 1 & 105 & 2 & 0.065 \\
\hline $\mathrm{Mg}(\mathrm{mg} / \mathrm{MJ})$ & $35 \cdot 7$ & 0.5 & 34.7 & 0.2 & $34 \cdot 2$ & 0.3 & 0.024 \\
\hline$\beta$-Carotene (mg/MJ) & 0.23 & 0.02 & 0.24 & 0.01 & 0.27 & 0.02 & $0.231^{*}$ \\
\hline Ascorbic acid (mg/MJ) & 7.55 & 0.44 & $8 \cdot 18$ & 0.21 & $8 \cdot 21$ & 0.33 & $0.087^{\star}$ \\
\hline Folate $(\mu \mathrm{g} / \mathrm{MJ})$ & $22 \cdot 8$ & 0.5 & $23 \cdot 1$ & 0.2 & 23.0 & 0.4 & $0.625^{\star}$ \\
\hline Vitamin E (mg/MJ) & 1.00 & 0.03 & 1.06 & 0.01 & 1.05 & 0.02 & $0.137^{*}$ \\
\hline \multicolumn{8}{|l|}{ Women } \\
\hline Subjects $(n)$ & \multicolumn{2}{|c|}{64} & \multicolumn{2}{|c|}{623} & \multicolumn{2}{|c|}{337} & \\
\hline Total energy intake (MJ) & $8 \cdot 2$ & 0.2 & $8 \cdot 8$ & 0.1 & $9 \cdot 1$ & 0.1 & $<0.0001$ \\
\hline Fat (\% of non-alcohol energy) & $42 \cdot 3$ & 0.7 & 39.9 & 0.2 & 38.4 & 0.3 & $<0.0001$ \\
\hline Carbohydrates (\% of non-alcohol energy) & $41 \cdot 3$ & 0.7 & 44.5 & 0.2 & $46 \cdot 2$ & 0.3 & $<0.0001$ \\
\hline Protein (\% of non-alcohol energy) & $16 \cdot 3$ & 0.3 & $15 \cdot 6$ & 0.1 & 15.4 & 0.1 & 0.011 \\
\hline Alcohol (\% of total energy) & $4 \cdot 3$ & 0.4 & 2.9 & $0 \cdot 1$ & $2 \cdot 2$ & 0.2 & $<0.0001$ \\
\hline Fibre (g/MJ) & 1.83 & 0.07 & $2 \cdot 12$ & 0.02 & $2 \cdot 28$ & 0.03 & $<0.0001^{*}$ \\
\hline $\mathrm{Fe}(\mathrm{mg} / \mathrm{MJ})$ & 1.50 & 0.04 & 1.60 & 0.01 & 1.62 & 0.02 & 0.009 \\
\hline $\mathrm{Ca}(\mathrm{mg} / \mathrm{MJ})$ & 129 & 4 & 126 & 1 & 126 & 2 & 0.715 \\
\hline $\mathrm{Mg}(\mathrm{mg} / \mathrm{MJ})$ & $36 \cdot 2$ & 0.7 & $36 \cdot 6$ & 0.2 & $37 \cdot 3$ & 0.3 & $0 \cdot 120$ \\
\hline$\beta$-Carotene (mg/MJ) & 0.36 & 0.04 & 0.39 & 0.01 & 0.42 & 0.02 & $0.507^{\star}$ \\
\hline Ascorbic acid (mg/MJ) & $10 \cdot 1$ & 0.8 & 11.9 & 0.2 & $12 \cdot 7$ & 0.3 & $<0.001^{*}$ \\
\hline Folate $(\mu \mathrm{g} / \mathrm{MJ})$ & $25 \cdot 0$ & 0.9 & $26 \cdot 2$ & 0.3 & $27 \cdot 4$ & 0.4 & $<0.001^{*}$ \\
\hline Vitamin E (mg/MJ) & $1 \cdot 10$ & 0.04 & $1 \cdot 15$ & 0.01 & $1 \cdot 13$ & 0.02 & $0.443^{*}$ \\
\hline
\end{tabular}

* Significance level tested with log-transformed variables.

† Misreporters of energy intake and subjects reporting past food habit change are excluded from the analysis.

past food habit change. Total EI (MJ), E\% from carbohydrates, and relative fibre intake increased significantly with the daily eating frequency, while E\% from fat, protein, and alcohol decreased. E\% from fat did not differ significantly for men with different eating frequency. Nutrient densities of ascorbic acid, folate and $\mathrm{Fe}$ were highest for women reporting six or more meals per $\mathrm{d}$. In men, nutrient density of $\mathrm{Mg}$ was higher for subjects with lower daily eating frequency (three or fewer and/or four to five meals per d). There were no significant differences in intakes of the various food groups apart from a higher intake of cake and pastries for women with a high eating frequency $(P=0 \cdot 036)$.

Table 4. BMI and waist circumference (WC) among men ( $n$ 892) and women ( $n$ 1024) in categories of daily eating frequency adjusting for potential confounders*

(Mean values and $95 \%$ confidence intervals)

\begin{tabular}{|c|c|c|c|c|c|c|c|}
\hline & \multicolumn{6}{|c|}{ Daily eating frequency } & \multirow[b]{3}{*}{$P$} \\
\hline & \multicolumn{2}{|c|}{3 or fewer } & \multicolumn{2}{|c|}{4 to 5} & \multicolumn{2}{|c|}{6 or more } & \\
\hline & Mean & $95 \% \mathrm{Cl}$ & Mean & $95 \% \mathrm{Cl}$ & Mean & $95 \% \mathrm{Cl}$ & \\
\hline \multicolumn{8}{|l|}{ Men } \\
\hline BMI $\left(\mathrm{kg} / \mathrm{m}^{2}\right)$ & $26 \cdot 7$ & $26 \cdot 0,27 \cdot 4$ & $26 \cdot 1$ & $25 \cdot 6,26 \cdot 5$ & $26 \cdot 0$ & $25 \cdot 4,26 \cdot 6$ & 0.158 \\
\hline WC $(\mathrm{cm})$ & $95 \cdot 2$ & $93 \cdot 2,97 \cdot 3$ & 93.5 & $92 \cdot 2,94 \cdot 8$ & $92 \cdot 7$ & $91 \cdot 0,94 \cdot 3$ & 0.088 \\
\hline \multicolumn{8}{|l|}{ Women } \\
\hline BMI $\left(\mathrm{kg} / \mathrm{m}^{2}\right)$ & $25 \cdot 2$ & $24 \cdot 2,26 \cdot 2$ & $25 \cdot 0$ & $24 \cdot 5,25 \cdot 6$ & $25 \cdot 0$ & $24 \cdot 3,25 \cdot 6$ & 0.903 \\
\hline$W C(\mathrm{~cm})$ & 78.5 & $75 \cdot 9,81 \cdot 1$ & $76 \cdot 1$ & $74 \cdot 7,77 \cdot 5$ & 75.9 & $74 \cdot 3,77 \cdot 6$ & 0.178 \\
\hline
\end{tabular}


Table 5. Associations between overweight, obesity, and moderate or severe central obesity (estimated separately) and daily eating frequency in men $(n$ 892)*

(Odds ratios and $95 \%$ confidence intervals)

\begin{tabular}{|c|c|c|c|c|c|c|c|c|c|c|}
\hline & \multirow[b]{2}{*}{ Subjects $(n)$} & \multicolumn{3}{|c|}{ Model 1† } & \multicolumn{3}{|c|}{ Model $2 \ddagger$} & \multicolumn{3}{|c|}{ Model $3 \S$} \\
\hline & & OR & $95 \% \mathrm{Cl}$ & $P$ for trend & OR & $95 \% \mathrm{Cl}$ & $P$ for trend & OR & $95 \% \mathrm{Cl}$ & $P$ for trend \\
\hline BMI 25 to $<30 \mathrm{~kg} / \mathrm{m}^{2} \|$ & & & & 0.919 & & & 0.867 & & & 0.818 \\
\hline 3 or fewer & 58 & 0.97 & $0.57,1.66$ & & 0.96 & $0.56,1.64$ & & 0.94 & $0.54,1.62$ & \\
\hline 4 to 5 & 262 & 0.85 & $0.59,1.21$ & & 0.84 & $0.59,1.21$ & & 0.85 & $0.59,1.22$ & \\
\hline 6 or more & 116 & 1.00 & & & 1.00 & & & 1.00 & & \\
\hline $\mathrm{BMI} \geq 30 \mathrm{~kg} / \mathrm{m}^{2} \|$ & & & & 0.037 & & & 0.039 & & & 0.044 \\
\hline 3 or fewer & 25 & $2 \cdot 45$ & $1 \cdot 06,5 \cdot 71$ & & 2.47 & $1 \cdot 05,5 \cdot 82$ & & $2 \cdot 42$ & $1.02,5.73$ & \\
\hline 4 to 5 & 67 & $1 \cdot 15$ & $0.60,2 \cdot 20$ & & $1 \cdot 15$ & $0.59,2.21$ & & $1 \cdot 15$ & $0.59,2.22$ & \\
\hline 6 or more & 20 & 1.00 & & & 1.00 & & & 1.00 & & \\
\hline WC 94 to $<102 \mathrm{~cm}$ ? & & & & 0.886 & & & 0.960 & & & 0.878 \\
\hline 3 or fewer & 36 & 1.04 & $0.60,1.80$ & & 0.99 & $0.57,1.72$ & & 1.05 & $0.59,1.84$ & \\
\hline 4 to 5 & 136 & 0.77 & $0.53,1.12$ & & 0.74 & $0.51,1.09$ & & 0.77 & $0.53,1.13$ & \\
\hline 6 or more & 67 & 1.00 & & & 1.00 & & & 1.00 & & \\
\hline$W C \geq 102 \mathrm{~cm}$ & & & & 0.029 & & & 0.028 & & & 0.043 \\
\hline 3 or fewer & 27 & $2 \cdot 18$ & $1.08,4.37$ & & $2 \cdot 21$ & $1.09,4.48$ & & 2.09 & $1.03,4.27$ & \\
\hline 4 to 5 & 102 & 1.67 & $0.99,2.80$ & & 1.64 & $0.97,2.77$ & & 1.64 & $0.97,2.78$ & \\
\hline 6 or more & 26 & 1.00 & & & 1.00 & & & 1.00 & & \\
\hline
\end{tabular}

* Misreporters of energy intake and subjects reporting past food habit change are excluded from the analysis.

† Model adjusted for age, education, socio-economic status, smoking, alcohol consumption, leisure-time physical activity, and total energy intake.

$\ddagger$ Model adjusted for age, education, socio-economic status, smoking, alcohol consumption, leisure-time physical activity, total energy intake, non-alcohol energy percentage from fat and fibre intake.

$\S$ Model adjusted for age, education, socio-economic status, smoking, alcohol consumption, leisure-time physical activity, total energy intake, non-alcohol energy percentage from fat, fibre intake, fruit and vegetables, confectionery foods, soft drinks, cakes and pastries and low-fat meat and low-fat dairy products.

\| Normal-weight (BMI 18.5 to $<25 \mathrm{~kg} / \mathrm{m}^{2}$ ) men $(n 338)$ were used as the reference.

I Men with a waist circumference less than $94 \mathrm{~cm}(n$ 496) were used as the reference.

The associations between daily eating frequency and the different overweight/obesity variables are shown in Tables 4-6. Mean BMI and waist circumference among men and women across daily eating frequency did not differ significantly when adjusting for potential confounders, and excluding subjects who were classified as misreporters of EI and reported a change of food habits in the past. The regression model adjusting for lifestyle confounders including total EI (Table 5, model 1) showed that men with a low daily eating frequency were more likely to be obese

Table 6. Associations between overweight, obesity, and moderate or severe central obesity (estimated separately) and daily eating frequency in women $(n$ 1024)*

(Odds ratios and $95 \%$ confidence intervals)

\begin{tabular}{|c|c|c|c|c|c|c|c|c|c|c|}
\hline & \multirow[b]{2}{*}{ Subjects $(n)$} & \multicolumn{3}{|c|}{ Model 1† } & \multicolumn{3}{|c|}{ Model $2 \ddagger$} & \multicolumn{3}{|c|}{ Model $3 \S$} \\
\hline & & OR & $95 \% \mathrm{Cl}$ & $P$ for trend & OR & $95 \% \mathrm{Cl}$ & $P$ for trend & OR & $95 \% \mathrm{Cl}$ & $P$ for trend \\
\hline BMI 25 to $<30 \mathrm{~kg} / \mathrm{m}^{2} \|$ & & & & 0.409 & & & 0.480 & & & 0.460 \\
\hline 3 or fewer & 16 & 0.74 & $0.37,1.51$ & & 0.77 & $0.38,1.58$ & & 0.76 & $0.37,1.57$ & \\
\hline 4 to 5 & 201 & 0.81 & $0.59,1.10$ & & 0.82 & $0.60,1.12$ & & 0.82 & $0.60,1.13$ & \\
\hline 6 or more & 126 & 1.00 & & & 1.00 & & & 1.00 & & \\
\hline $\mathrm{BMI} \geq 30 \mathrm{~kg} / \mathrm{m}^{2} \|$ & & & & $0 \cdot 118$ & & & 0.080 & & & 0.069 \\
\hline 3 or fewer & 9 & $2 \cdot 18$ & $0 \cdot 82,5 \cdot 78$ & & 2.45 & $0.90,6.67$ & & 2.55 & $0.93,6.98$ & \\
\hline 4 to 5 & 73 & $1 \cdot 20$ & $0.74,1.93$ & & $1 \cdot 24$ & $0.77,2 \cdot 01$ & & $1 \cdot 26$ & $0.77,2.05$ & \\
\hline 6 or more & 36 & 1.00 & & & 1.00 & & & 1.00 & & \\
\hline WC 80 to $<88 \mathrm{~cm} \emptyset$ & & & & 0.431 & & & 0.292 & & & 0.232 \\
\hline 3 or fewer & 14 & $1 \cdot 41$ & $0.60,3 \cdot 28$ & & 1.59 & $0.67,3.79$ & & $1 \cdot 70$ & $0.71,4.08$ & \\
\hline 4 to 5 & 106 & 0.85 & $0.58,1.24$ & & 0.89 & $0.60,1 \cdot 31$ & & 0.91 & $0.62,1.35$ & \\
\hline 6 or more & 65 & 1.00 & & & 1.00 & & & 1.00 & & \\
\hline$W C \geq 88 \mathrm{~cm} \|$ & & & & 0.072 & & & 0.121 & & & 0.115 \\
\hline 3 or fewer & 12 & $2 \cdot 17$ & $0.93,5.05$ & & 1.98 & $0.84,4.69$ & & $2 \cdot 01$ & $0.84,4.79$ & \\
\hline 4 to 5 & 79 & 1.09 & $0.71,1.66$ & & 1.06 & $0.69,1.63$ & & 1.06 & $0.70,1.66$ & \\
\hline 6 or more & 43 & 1.00 & & & 1.00 & & & 1.00 & & \\
\hline
\end{tabular}

* Misreporters of energy intake and subjects reporting past food habit change are excluded from the analysis.

† Model adjusted for age, education, socio-economic status, smoking, alcohol consumption, leisure-time physical activity and total energy intake.

$\ddagger$ Model adjusted for age, education, socio-economic status, smoking, alcohol consumption, leisure-time physical activity, total energy intake, non-alcohol energy percentage from fat and fibre intake.

$\S$ Model adjusted for age, education, socio-economic status, smoking, alcohol consumption, leisure-time physical activity, total energy intake, non-alcohol energy percentage from fat, fibre intake, fruit and vegetables, confectionery foods, soft drinks, cakes and pastries and low-fat meat and low-fat dairy products.

$\|$ Normal-weight (BMI 18.5 to $<25 \mathrm{~kg} / \mathrm{m}^{2}$ ) women $(n 546)$ were used as the reference.

I Women with a waist circumference less than $80 \mathrm{~cm}(n 704)$ were used as the reference. 
(i.e. $\mathrm{BMI} \geq 30 \mathrm{~kg} / \mathrm{m}^{2} ; P$ for trend $=0.037$ ) and have a large waist circumference (i.e. $\geq 102 \mathrm{~cm} ; P$ for trend=0.029) compared with men with a high daily eating frequency (i.e. six or more). Results in women failed to reach statistical significance. The daily eating frequency was not significantly associated with the likelihood of being overweight or having a waist circumference of 94 to $<102 \mathrm{~cm}$ in men or a waist circumference of 80 to $<88 \mathrm{~cm}$ in women. The interaction between obesity, eating frequency, and sex was non-significant.

Several regression models were considered in order to adjust for diet composition. However, the inclusion of both fat and carbohydrate densities was the only combination that attenuated results to the degree of borderline significance (results not shown). However, the strong correlation (correlation coefficient $=0.94$ ) between fat and carbohydrate intake $(\mathrm{E} \%)$ indicates that including both in the model might be superfluous. Adjusting for various food groups thought to be important in the development of obesity (including dietary fibre, fruit and vegetables, confectionery foods, soft drinks, and cakes and pastries, high- or low-fat meat, and high- or low-fat dairy) attenuated the results. However, men with a low eating frequency still had an increased risk of general $(P=0 \cdot 044)$ and central obesity $(P=0 \cdot 043)$ (Table 5, model 3$)$.

\section{Discussion}

The results suggest that a high daily eating frequency is associated with an overall healthy lifestyle in both men and women. A high daily eating frequency was associated with higher leisure-time physical activity, non-smoking, lower alcohol consumption, and lower $\mathrm{E} \%$ from fat and alcohol and higher $\mathrm{E} \%$ from carbohydrates and higher relative fibre intake. Men with a low eating frequency showed an increased risk of general and central obesity, which remained when adjusting for lifestyle and dietary factors. Results for women showed similar but non-significant tendencies.

Participants in the MDC cohort are in many aspects representative of the source population; differences between participants and non-participants in the MDC study have been described elsewhere ${ }^{(31)}$. A common source of bias in studies on diet and obesity is misreporting of food intake. 'Social desirability' may influence both what subjects actually eat and what they report eating. Obese individuals tend to under-report their dietary intake ${ }^{(42-45)}$, but whether the type of under-reporting in the present study is selective under-reporting of certain foods or general under-reporting of all foods is difficult to determine. Cross-sectional studies in particular are often hindered by the effects of misreporting by subjects as well as change in food habits over time. The present study attempted to overcome these potential sources of bias by excluding both energy misreporters and subjects reporting to have changed their food habits substantially in the past. Excluding these individuals reduces the likelihood of including those prone to under-report eating occasions as well as those with restrained eating. In addition, the eating frequency was defined as all eating occasions during an ordinary day (including both meals and snacks), which, together with the careful checking of self-reported diet information during interview, reduces the likelihood of misclassification of eating frequency.

An important finding is that the daily eating frequency was linked to differences in dietary quality. A high daily eating frequency was associated with higher E\% from carbohydrates and relative intake of fibre, and lower $\mathrm{E} \%$ from protein and fat. Individuals with a high daily eating frequency may be more likely to consume healthy snacks (for example, sandwiches and fruits) in between main meals (i.e. cooked meals). The high relative fibre intake together with higher density of ascorbic acid and folate also suggest a higher consumption of fruit and vegetables for women with a higher daily eating frequency. A higher $\mathrm{E} \%$ from protein may indicate consumption of primarily cooked meals (including meat) for those reporting a low daily eating frequency.

The present study suggests that there may be a favourable impact of increasing eating frequency in regards to preventing general and central obesity. Although the associations seen among men in the present study (Table 5) remained significant when adjusting for lifestyle and dietary confounders (including total EI), it is possible that residual confounding of both lifestyle factors and/or energy or dietary intake could contribute to the results. Thus, the present results may rather indicate that individuals with a more active lifestyle, resulting in higher energy expenditure and intake, are likely to consume a more 'healthy' diet, which results in reduced body fatness and waist circumference. In addition, the cross-sectional design makes it impossible to determine if reverse causation (i.e. obese individuals eat fewer meals per $\mathrm{d}$ as a strategy for weight loss) is an underlying factor. A previous study within the MDC cohort suggests that individuals (especially men) with fibre-rich food patterns are less likely to be centrally obese ${ }^{(46)}$. In the present study a high fibre intake was the clearest diet-quality indicator associated with a high eating frequency among men, and thus, consistent with the study by Wirfält et al. ${ }^{(46)}$, likely to predict a healthy waist circumference. However, in the present study it is difficult to determine if a high daily eating frequency in itself has beneficial health effects. Evidence is inconclusive in the literature regarding a possible metabolic effect of eating frequency. It has previously been hypothesised that a high eating frequency has an impact on appetite control, and improves factors such as dietary induced thermogenesis, lipidaemia and glycaemia ${ }^{(47-49)}$. In addition, a study by Titan et al. ${ }^{(50)}$ showed an inverse relationship between serum lipid concentrations and eating frequency in a freeliving population. A plausible explanation for the observed sex differences in the present study is a low discriminating power due to very few women reporting three or fewer meals per $d$. The association with a high fibre intake also stresses the possible benefits of high-fibre food (including fruit and vegetables).

In conclusion, the present study suggests that a high eating frequency in the context of an overall healthy lifestyle and a higher intake of low-fat and high-fibre foods may be inversely associated with the likelihood of general and central obesity in middle-aged men. Since body-fat mass, as well as the distribution of body fat, are risk factors of diabetes, CVD and several forms of cancer ${ }^{(51-53)}$, our findings should be of interest to public health professionals involved in chronic disease prevention. Because the present cross-sectional study cannot differentiate cause from effect, the observed association between eating frequency, diet and body composition warrants further investigation, particularly prospective research and intervention studies. 


\section{Acknowledgements}

The present investigation was supported by the Ernhold Lundström Foundation, Lund University and Region Skåne.

I. $H$. conducted the study and wrote the manuscript. All authors contributed to the interpretation of the results and to the finalisation of the manuscript.

There are no conflicts of interest.

\section{References}

1. Martínez-González M, Martinéz J, Hu F, et al. (1999) Physical inactivity, sedentary lifestyle and obesity in the European Union. Int J Obes Relat Metab Disord 11, 1192-1201.

2. Ball K, Owen N, Salmon J, et al. (2001) Associations of physical activity with body weight and fat in men and women. Int $J$ Obes 25, 914-919.

3. Lindström M, Isacsson S \& Merlo J (2003) Increasing prevalence of overweight, obesity and physical inactivity. Eur $J$ Public Health 12, 306-312.

4. Koh-Banerjee P, Chu N, Spiegelman D, et al. (2003) Prospective study of the association of changes in dietary intake, physical activity, alcohol consumption, and smoking with 9-y gain in waist circumference among 16587 US men. Am J Clin Nutr 78, 719-727.

5. Lissner L, Johansson S, Qvist J, et al. (2000) Social mapping of the obesity epidemic in Sweden. Int J Obes 24, 801-805.

6. Berg C, Rosengren A, Aires N, et al. (2005) Trends in overweight and obesity from 1985 to 2002 in Göteborg, West Sweden. Int J Obes 29, 916-924.

7. Ogden C, Carroll M, Curtin L, et al. (2006) Prevalence of overweight and obesity in the United States, 1999-2004. JAMA 295, 1549-1555.

8. Sanchez A, Norman G, Sallis J, et al. (2008) Patterns and correlates of multiple risk behaviours in overweight women. Prev Med 46, 196-202.

9. Newby P, Muller D, Hallfrisch J, et al. (2003) Dietary patterns and changes in body mass index and waist circumference in adults. Am J Clin Nutr 77, 1417-1425.

10. Schulze M, Fung T, Manson J, et al. (2006) Dietary patterns and changes in body weight in women. Obesity 14, 1444-1453.

11. McAllister E, Dhurandhar N, Keith S, et al. (2009) Ten putative contributors to the obesity epidemic. Crit Rev Food Sci Nutr 49, 868-913.

12. Swinburn B, Caterson I, Seidell J, et al. (2004) Diet, nutrition and the prevention of excess weight gain and obesity. Public Health Nutr 7, 123-146.

13. Drummond S, Crombie N, Cursiter M, et al. (1998) Evidence that eating frequency is inversely related to body weight status in male, but not female, non-obese adults reporting valid dietary intakes. Int J Obes 22, 105-112.

14. Bertéus Forslund H, Torgerson J, Sjöström L, et al. (2005) Snacking frequency in relation to energy intake and food choices in obese men and women compared to a reference population. Int J Obes 29, 711-719.

15. Toschke A, Küchenhoff H, Koletzko B, et al. (2005) Meal frequency and childhood obesity. Obes Res 13, 1932-1938.

16. Fung T, Rimm E, Spiegelman D, et al. (2001) Association between dietary patterns and plasma biomarkers of obesity and cardiovascular disease risk. Am J Clin Nutr 73, 61-67.

17. Fábry P, Fodor J, Geizerová H, et al. (1968) Meal frequency and ischaemic heart disease. Lancet ii, 190-191.

18. Bellisle F, McDevitt R \& Prentice A (1997) Meal frequency and energy balance. Br J Nutr 77, Suppl. 1, S57-S70.
19. Hampl J, Heaton C \& Taylor C (2003) Snacking patterns influence energy and nutrient intakes but not body mass index. J Hum Nutr Diet 16, 3-11.

20. Ma Y, Bertone E, Stanek E III, et al. (2003) Association between eating patterns and obesity in a free-living US adult population. Am J Epidemiol 158, 85-92.

21. Davis J, Hodges V \& Gillham B (2006) Normal-weight adults consume more fiber and fruit than their age- and height-matched overweight/obese counterparts. J Am Diet Assoc 106, 833-840.

22. Greenwood J \& Stanford J (2008) Preventing or improving obesity by addressing specific eating patterns. J Am Board Fam Med 21, 135-140.

23. Kushner RF \& Choi S (2009) Prevalence of unhealthy lifestyle patterns among overweight and obese adults. Obesity (Silver Spring) (epublication ahead of print version 29 October 2009).

24. Westenhoefer J (2005) Age and gender dependent profile of food choice. Forum Nutr 57, 44-51.

25. Neumark-Sztainer D, Sherwood N, French S, et al. (1999) Weight control behaviors among adult men and women: cause for concern? Obes Res 7, 179-188.

26. Maurer J, Taren D, Teixeira P, et al. (2006) The psychosocial and behavioral characteristics related to energy misreporting. Nutr Rev 64, 53-66.

27. Mattisson I, Wirfält E, Aronsson C, et al. (2005) Misreporting of energy: prevalence, characteristics of misreporters and influence on observed risk estimates in the Malmö Diet and Cancer cohort. Br J Nutr 94, 832-842.

28. Sonestedt E, Wirfält E, Gullberg B, et al. (2005) Past food habit change is related to obesity, lifestyle and socio-economic factors in the Malmo Diet and Cancer Cohort. Public Health Nutr 8, $876-885$.

29. Sonestedt E, Gullberg B \& Wirfält E (2007) Both food habit change in the past and obesity status may influence the association between dietary factors and postmenopausal breast cancer. Public Health Nutr 10, 769-779.

30. Berglund G, Elmståhl S, Janzon L, et al. (1993) The Malmö Diet and Cancer study. Design and feasibility. J Intern Med 233, 53-57.

31. Manjer J, Carlsson S, Elmstahl S, et al. (2001) The Malmo Diet and Cancer Study: representativity, cancer incidence and mortality in participants and non-participants. Eur J Cancer Prevent 10, 489-499.

32. Callmer E, Riboli E, Saracci R, et al. (1993) Dietary assessment methods evaluated in the Malmo Food Study. J Intern Med 233, $53-57$.

33. Elmståhl S, Riboli E, Lindegärde F, et al. (1996) The Malmö Food Study: the relative validity of a modified diet history method and an extensive food frequency questionnaire for measuring food intake. Eur J Clin Nutr 50, 143-151.

34. Riboli E, Elmståhl S, Saracci R, et al. (1997) The Malmö Food Study: validity of two dietary assessment methods for measuring nutrient intake. Int $J$ Epidemiol 26, Suppl. 1, S161-S173.

35. Elmståhl S, Gullberg B, Riboli E, et al. (1996) The Malmö Food Study: the reproducibility of a novel diet history method and an extensive food frequency questionnaire. Eur J Clin Nutr 50, $134-142$.

36. Goldberg G, Black A, Jebb S, et al. (1991) Critical evaluation of energy intake using fundamental principles of energy physiology: 1. Derivation of cut-off limits to identify underrecording. Eur J Clin Nutr 45, 569-581.

37. Black A (2000) Critical evaluation of energy intake using the Goldberg cut-off for energy intake: basal metabolic rate. A practical guide to its calculation, use and limitations. Int $J$ Obes Relat Metab Disord 24, 1119-1130.

38. National Bureau of Statistics (1989) Occupations in Population and Housing Census 1985 (FoB 85) according to Nordic 
standard occupational classification (Nordisk yrkesklassificering NYK) and Swedish socioeconomic classification (Socioekonomisk indelning SEI) (article in Swedish). Stockholm: Statistics Sweden.

39. Taylor H, Jacobs D, Schucker B, et al. (1978) A questionnaire for the assessment of leisure time physical activities. J Chronic Dis 31, 741-755.

40. Richardson M, Leon A, Jacobs D, et al. (1994) Comprehensive evaluation of the Minnesota Leisure Time Physical Activity Questionnaire. J Clin Epidemiol 47, 271-281.

41. World Health Organization (2000) Obesity: Preventing and Managing the Global Epidemic. Report on a WHO Consultation. WHO Technical Report Series, no. 894. Geneva: WHO.

42. Lafay L, Basdevant A, Charles M, et al. (1997) Determinants and nature of dietary underreporting in a free-living population: the Fleurbaix Laventie Ville Santé (FLVS) Study. Int J Obes Relat Metab Disord 7, 567-573.

43. Braam L, Ocké M, Bueno-de-Mesquita H, et al. (1998) Determinants of obesity-related underreporting of energy intake. Am J Epidemiol 147, 1081-1086.

44. Bazelmans C, Matthys C, De Henauw S, et al. (2007) Predictors of misreporting in an elderly population: the 'Quality of Life after 65' study. Public Health Nutr 10, 185-191.

45. Heitmann B \& Lissner L (1995) Dietary underreporting by obese individuals - is it specific or non-specific? BMJ 311, 986-989.

\section{Appendix}

Presented is an example of how the meal order during a typical week was self-reported by the participants in the Malmö Diet and Cancer study, including instructions to participants. The daily eating frequency was calculated from the total number of meals per week.
46. Wirfält E, Hedblad B, Gullberg B, et al. (2001) Food patterns and components of the metabolic syndrome in men and women: a cross-sectional study within the Malmö Diet and Cancer cohort. Am J Epidemiol 154, 1150-1159.

47. Farshchi H, Taylor M \& Macdonal I (2005) Beneficial metabolic effects of regular meal frequency on dietary thermogenesis, insulin sensitivity, and fasting lipid profiles in healthy obese women. Am J Clin Nutr 81, 16-24.

48. Jenkins D, Wolever T, Vuksan V, et al. (1989) Nibbling versus gorging: metabolic advantages of increased meal frequency. $N$ Engl J Med 321, 929-934.

49. LeBlanc J, Mercier I \& Nadeau A (1993) Components of postprandial thermogenesis in relation to meal frequency in humans. Can J Physiol Pharmacol 71, 879-883.

50. Titan SM, Bingham S, Welch A, et al. (2001) Frequency of eating and concentrations of serum cholesterol in the Norfolk population of the European Prospective Investigation into Cancer (EPIC-Norfolk): cross sectional study. BMJ 323, 1286.

51. Hu F (2003) Overweight and obesity in women: health risks and consequences. J Womens Health (Larchmt) 12, 163-172.

52. Flegal K (2005) Epidemiologic aspects of overweight and obesity in the United States. Physiol Behav 86, 599-602.

53. Abu-Abid S, Szold A \& Lausner J (2002) Obesity and cancer. J Med 33, 73-86.

\section{Meal intake}

Describe in broad terms the meals including snacks that you usually eat/drink during one day. Note the name of the meal, the time of the meal, what it consists of, and how many times per week you usually eat this meal. If Saturday and Sunday are significantly different from weekdays, you can report two meal orders.

For an example, see the Table below.

\begin{tabular}{llll}
\hline Meal name & Time (approximate) & What does it consist of? & How many times per week? \\
\hline Monday to Friday & & & \\
$\quad$ Breakfast & 06.30 hours & Coffee + two sandwiches & 5 \\
Before lunch & 10.00 hours & Fruit or biscuit with tea & 5 \\
Lunch & 12.00 hours & Cooked meal, bread, beer & 5 \\
Afternoon coffee & 14.00 hours & Coffee + sweet roll & 5 \\
Dinner & 18.00 hours & Three or four sandwiches with different spreads & 5 \\
Evening tea & 20.00 hours & Tea + small cake (Friday night drink, cheese, or shrimps) & $4+1$ \\
Saturday and Sunday & & & 2 \\
Breakfast & 08.00 hours & Coffee, porridge, sandwich, juice & 2 \\
Morning coffee & 10.30 hours & Coffee + cake & 2 \\
Lunch & 12.00 hours & Sandwiches + beer & 2 \\
Afternoon coffee & 15.00 hours & Coffee + pastry & 2 \\
Dinner & 18.00 hours & Cooked meal, dessert, wine on Saturdays & 2 \\
Evening tea & 20.00 hours & Tea + biscuits & \\
\hline
\end{tabular}

